\title{
ON THE UNCERTAINTIES IN ASSESSING THE ATMOSPHERIC EFFECTS OF LAUNCHERS
}

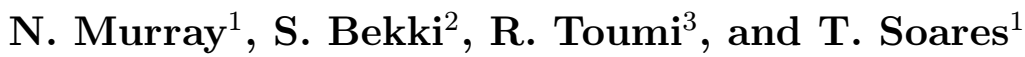 \\ ${ }^{1}$ European Space Agency, ESTEC \\ 1 Keplerlann, AG Noordwijk 2200, The Netherlands \\ ${ }^{2} \mathrm{UPMC}, \mathrm{CNRS}$ \\ 6 Place Jussieu, Paris 75005, France \\ ${ }^{3}$ Imperial College London \\ London SW7 2AZ, U.K.
}

\begin{abstract}
This paper discusses the areas contributing to uncertainty in the modeling of the effects of launchers on the atmosphere. The main areas dealt with are $(i)$ the aerothermodynamics of rocket plumes which includes the combustion and expansion process and ( $i i)$ climate modeling including the insertion of small scale plumes into relatively large scale climate models. The complexity of modeling necessary for a proper and accurate assessment of plume effects implies the need for validation data which may require flight and ground campaigns as well as laboratory experiments.
\end{abstract}

\section{INTRODUCTION}

The awareness of the environmental issues linked to human activities is continuously growing and it is translating into various initiatives, such as an increased regulation and a deeper scientific analysis of environmental aspects. The European Space Agency (ESA) is striving to determine the environmental impact of its space activities in line with its position on the sustainability and the environmental awareness including possible environmental impacts of launchers. It is generally considered in the rocket and atmospheric communities that due to the low frequency of launches worldwide, rocket emissions do not have a significant impact on the global environment. However, there still remains a need to quantify the actual emission impact per launch since, as a scientific agency, ESA wishes to continuously improve its understanding so as to be prepared to reply to questions on the topic and cannot rely on unsubstantiated claims.

The atmospheric impact has been considered previously in space missions involving ESA, an example being the Cassini-Huygens mission in 1997, an international cooperative scientific mission between National Aeronautics and Space Administration (NASA), ESA, and Agenzia Spaziale Italiana (ASI). Before launch,

This is an Open Access article distributed under the terms of the Creative Commons Attribution License 2.0, which permits unrestricted use, distribution, and reproduction in any medium, provided the original work is properly cited. 
a full Environmental Impact Statement (EIS) [1] was published, driven mainly by the concern over the radioisotope thermoelectric generators (RTGs) on board, these being nuclear reactors that convert the energy produced by the decay of plutonium into electricity using the Seebeck effect. As a part of this EIS, the impact of the rocket engines on the ground and in the atmosphere were evaluated using existing data coupled with numerical models, although, the authors do state that "The accuracy of the models is limited by the difficulty in modelling simultaneous and complex chemical reactions concurrently with three-dimensional stratospheric transport effects". This is qualified, however, with the implied acknowledgement that future work is required to validate assumptions and hypotheses.

The U.S. has been at the forefront of the research into this topic and has conducted several aircraft measurement campaigns through the plumes of its heavy and small launch vehicles (Titan IV, Athena II, Atlas II, and the Space Shuttle $[2-5])$. These measurements provided invaluable scientific data and insight into the effects of plumes on the environment. Specific outcomes were:

- up to $100 \%$ of the ozone in the plume region was depleted;

- the local ozone levels tended to recover to ambient levels after an order of hours;

- launch vehicles with very different fuel burn rates caused the same levels of ozone depletion in the first $60 \mathrm{~min}$ after the launch. This was contrary to the results of plume-wake models [6];

- afterburning in the plume affects the partition between ozone-destroying compounds such as chlorine radicals and reservoir species such as $\mathrm{HCl}$; and

- it is also worth pointing that water vapor in large rocket plumes can also be detected in the middle atmosphere from satellites [7] and has been linked to the formation of mesospheric clouds [8].

However, no firm conclusion can currently be derived on the global effects of launchers. The issue of the quantification of atmospheric impact is complex, requiring a complete knowledge of the aerothermodynamics of rocket plumes including the combustion, the expansion in the nozzle, and the development in the atmosphere. The small scale plume must then be integrated into relatively large scale climate models without loss in fidelity.

The further sections will discuss the points of uncertainty that exist when modeling the plume from the combustor and including its development in the atmosphere. Following this the integration of the plume into climate models will be analysed and the current uncertainties involved will be described. The list of points is not meant to be complete but to give an impression of the level of computational resources and complexity required. Finally, further work required to properly compute the atmospheric effects of launcher plumes will be described. 


\section{UNCERTAINTIES IN SIMULATING ROCKET PLUMES}

\subsection{Overview}

The chemical composition and conditions within a rocket plume depend on a number of factors that are linked to complex physical processes and phenomena. In the past, the plumes themselves have been of interest to the Agency in limited situations. Environmental aspects at CSG (Centre Spatial Guyanais) are monitored and measured since the very first Ariane launches. At the occasion of each launch a measurement campaign is put in place to monitor effects on the local flora and fauna. All measurements show that the environmental impacts are limited to the area of $1 \mathrm{~km}$ around a launch pad.

Plume contamination in terms of excess radiation to the payload and chemical contamination on the optical payload are of importance in extra atmosphere conditions and require coupled Navier-Stokes and DSMC (Direct Simulation Monte Carlo) (or similar) codes with multiphase physics models including the trajectory analysis of the solid alumina phase. The simulation of the plume evolution between 1 and $100 \mathrm{~km}$ is complex and requires careful consideration of various inter-dependent phenomena, as elucidated in the sections below.

\subsection{Processes Affecting the Conditions in the Early Plume}

The conditions of the plume close to the nozzle exit plane are sometimes approximated using equilibrium chemistry and ideal expansion assumptions for example. The plume development may similarly be approximated, but the actual conditions at the nozzle exit and in the developing plume are likely to differ significantly due to a number of complex phenomena. A schematic of a plume flowfield from Sutton [9] is shown in Fig. 1. The interaction with the external flowfield introduces a complex wave pattern which requires precise modeling since the conditions due to this interaction affect the chemistry of the plume. The following sections describe some of the phenomena associated with this interaction but the list is far from exhaustive.

\subsubsection{Afterburning}

Afterburning is generally noted in the literature as significantly modifying the early plume conditions. It occurs due to the recompression of the plume due to the interaction with the freestream (see Fig. 1) and is caused by the mismatch in pressure between the two streams. It increases the local temperature which, in turn, will affect the species concentrations in the plume. If the plume mixing process introduces oxygen into the usually fuel rich plume further combustion 
Thickness of mixing or afterburning layer increases with length

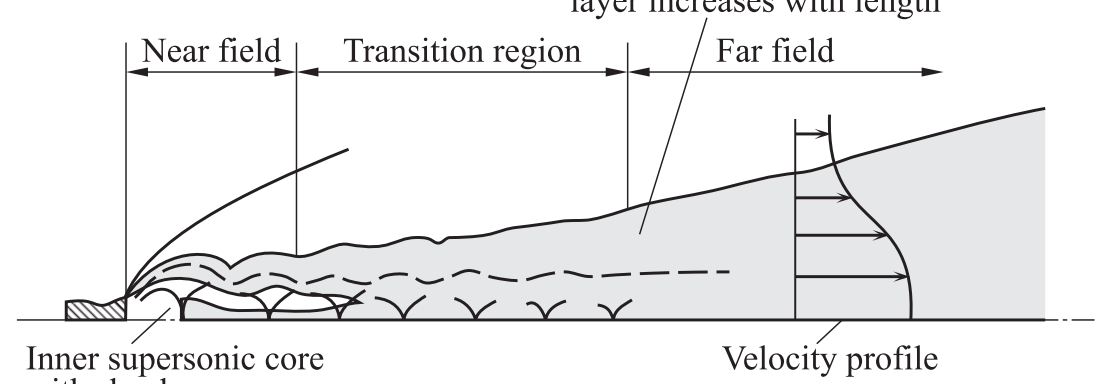

with shock waves

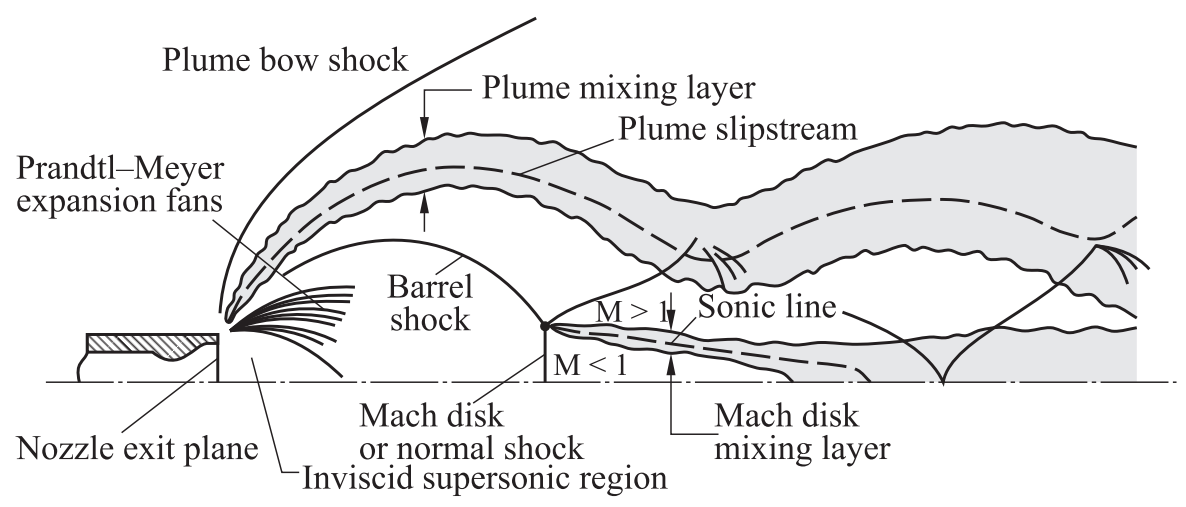

Figure 1 Plume schematic taken from [9]

and heat release will occur resulting in further chemistry and, in particular, partitioning of important plume species.

Therefore, chemical species fractions typically determined by computation at the nozzle exit plane are not comparable with the actual fractions measured in the early plume during U.S. flight campaigns. Modeling of afterburning, therefore, requires an accurate representation of the plume flowfield which, in turn, relies on the models and assumptions made during the combustion phase, nozzle expansion phase, and the plume development. Phenomena that affect the afterburning process are:

- the finite chemical kinetics that occur;

- the presence of particulate;

- mixing of the plume with freestream;

- the presence of boundary layers;

- the temporal freestream conditions; and

\section{4}


- the launcher configuration including the upstream topology and the rocket configuration.

These will all be discussed in further sections, but they all add to the uncertainty involved in the understanding and simulation of the afterburning process and must be accurately modeled if the temperatures in the early plume are to be reproduced in simulations.

\subsubsection{Finite kinetics}

Since the recent advancements in the computational power it has been possible to model the finite kinetics of combustion using reaction rates and constants, $k$, typically in the form of the Arrhenius equation as in

$$
\text { Rate }=k[B]^{b}[C]^{c}=A e^{-E /(R T)}[B]^{b}[C]^{c} .
$$

Here, $E$ is the activation energy; $T$ is the temperature; and $A$ is related to the frequency of molecular collisions.

The effect of using a finite rate scheme rather than an equilibrium chemistry assumption can be seen when assessing the conditions in the early nozzle just downstream of the throat where the flow rapidly expands. In this situation the density and temperature drop dramatically. As a result, the frequency and energy of collisions both drop sharply. As the flow expands in the nozzle, the density and temperature drop to a point where some reaction rates become negligible and flow species do not change, i.e., the flow is Frozen. However, an equilibrium calculation would allow for further reactions to occur. Frozen points can be artificially subjected to a point in the equilibrium calculation but this relies on estimation rather than real kinetics. Finite kinetics would also be required to assess the chemistry occurring in the mixing and afterburning regions (explained later).

It is, therefore, important that finite kinetic routines be used to assess the combustion process and the plume development. These routines should include heterogeneous reactions on particulates because the surfaces of particles, which will be discussed later, are often chemically reactive. They play an important role in the chemistry within the plume and also in the combustion chamber. There is the need for laboratory experiments to measure some key reaction constants (particularly for heterogeneous reactions) and to assess the full set of reaction mechanisms.

\subsubsection{Plume/freestream mixing}

As the plume develops it mixes with the freestream, entraining air into the plume core as in Fig. 1. The mixing rate depends on the conditions of the 
plume/freestream interface. Typical factors affecting the mixing layer of a jet in co-flow (equivalent to the rocket plume situation) are:

- laminar/turbulent interface - the shear stresses in a turbulent shear layer can be of the order of 100's of times greater than those in laminar shear layer [10]. The turbulent kinetic energy increases momentum and thermal mixing dramatically and can, therefore, help to dilute the plume and lower ozone depletion;

- convective Mach number - there is some dispute as to whether or not the convective Mach number is the best correlation with the shear layer mixing but it is acknowledged that the mixing level drops (relative to the incompressible value) as the convective Mach number increases. This phenomenon is illustrated in Fig. 2 and clearly demonstrates the sensitivity of the shear layer development to the convective Mach number, particularly when $0<\mathrm{M}_{c}<1$; and

- density and velocity ratio - Gutmark et al. [12] showed that the mixing layer development depended on the velocity and density ratio and, in the conditions he considered, increased as the velocity difference in the shear layer increased and as the relative momentum of the slower layer increased.

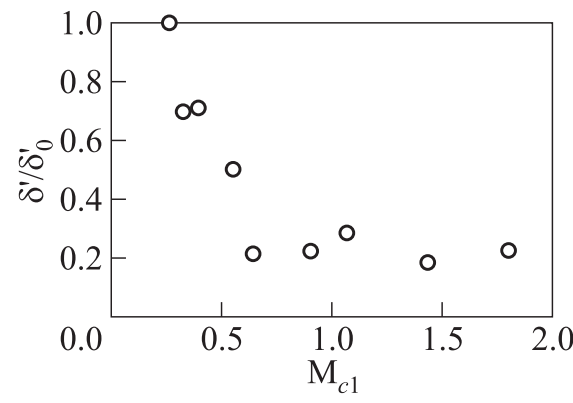

Figure 2 Normalised shear layer development vs. convective Mach number [11]

Whilst these facts are known with some degree of confidence, CFD (Computational Fluid Dynamics) codes do not necessarily capture their effects on mixing correctly, particularly when the flow is turbulent. It should be noted that compressibility effects as a whole (Convective Mach number is a good example) require significant validation in turbulent boundary layers when using RANS (Reynolds-Averaged NavierStokes) type solvers. Due to the assumptions made, the turbulence model can neglect some of the important compressibility features of turbulence in shear layers. Tsimis [10] found that a number of turbulence models did not capture the sensitivity of the shear layer mixing rate unless Heinza's compressibility correction [13] was implemented. Without the correction the shear layer was developing laterally faster than in his experiments. Since the ozone depletion in the plume depends on the mixing rate [14], any computations need to be carefully validated. Higher order computations such as LES (Large Eddy Simulation) or DNS (Direct Numerical Simulation) could of course be used, but 
certainly, for DNS and possibly for LES, the amount of computational resources required for a plume calculation would be prohibitive.

\subsubsection{Particulate}

Solid fuel combustion products contain large mass fractions of particulate, particularly alumina. The density of the alumina particulate is two orders of magnitude higher than the gaseous species mix, and as such does not expand due to the pressure gradients developed by the nozzle expansion contour. Instead the particulate reacts to the drag force created by relative velocity between its undisturbed velocity and the expanding flow. The drag force is typically $10^{5}-10^{7}$ times larger than the force produced by pressure gradients in the flow and can be assumed to be the only force acting on the particles. The drag interaction turns the trajectory of the particulate but also similarly turns the trajectory of the gas as in Fig. 3.

The change in the flowfield due to the particulate is important in the atmosphere as it exacerbates the afterburning effect. This is clearer in Fig. 4 where the plume flowfields calculated with and without particulate from Fig. 3 is shown (note that no chemistry was modeled). The presence of the alumina reduces the velocity, increases the local temperature and results in a separation region at the centreline at $X=8$. This further raises the local temperature significantly and increases residence times in the downstream subsonic region; both of which will promote further chemical reactions such as $\mathrm{Cl}$ formation.

The interaction between the flow and particulates is sensitive to the size distribution of the particles since smaller particles exert less force on the flow and follow the streamlines more than heavier particles. There is therefore a requirement for precise quantification of the particle number and distribution. This is

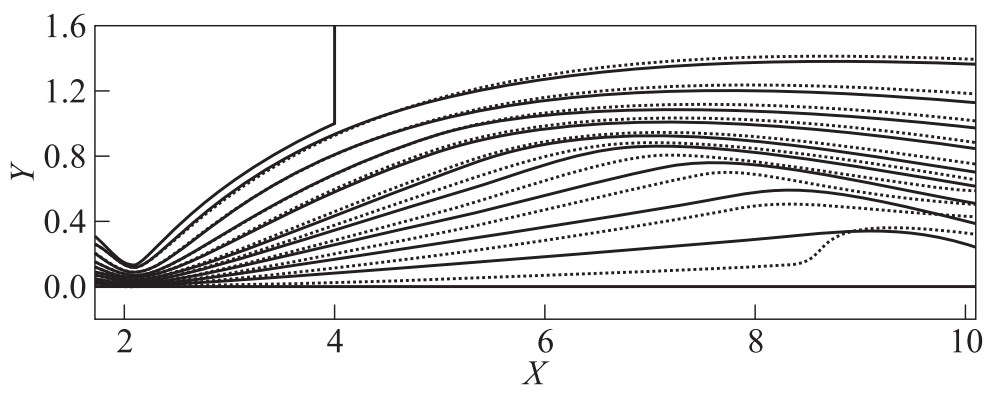

Figure 3 Streamlines in a nozzle and plume without particulate (solid curves) and with particulate (dotted curves). The presence of the high mass particulate decelerates the plume flowfield and results in a subsonic region at the plume centreline at $x=8$. Spatial coordinates are nondimensionalized by the nozzle exit radius [15] 

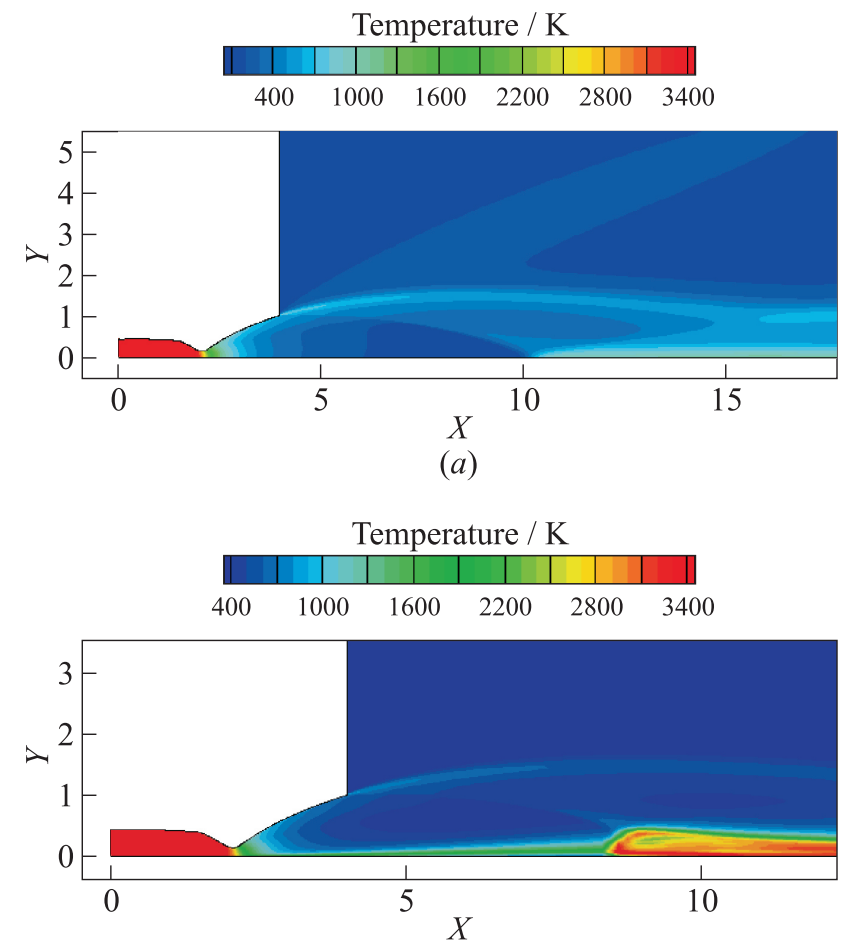

(b)

Figure 4 Comparison between plume flowfield without $(a)$ and with $(b)$ the alumina particulate. Spatial coordinates are nondimensionalized by the nozzle exit radius [15].

difficult to quantify and could be sensitive to not only the combustion process but also to other factors such as plume conditions. Ground testing is required where rocket test benches are instrumented with some means of quantifying the distribution. For example, since PIV (Particle Image Velocimetry) type techniques already sample particle distributions, one could imagine a very small step to use these apparatus to visualise the alumina particles in a simple rocket test bench.

Another important impact that alumina particulate may have on the plume chemical composition and conditions is its contribution to heterogeneous chemistry. Gas-phase chemistry cannot explain all the ozone losses seen in U.S. flight campaigns [16]. Therefore, this could indicate the presence of either heterogeneous chemistry on $\mathrm{Al}_{2} \mathrm{O}_{3}$ particles activating chlorine ozone-destroying radicals or even direct ozone loss on particles [17]. The uncertainties on the mechanisms, kinetics and rate constants of heterogeneous reactions on alumina surfaces are 
very large [18]. More laboratory work is required on reducing these uncertainties and on assessing the effect of particle surface aging on their chemical reactivity. Since a range of species is likely to stick to the particles, sticking probabilities are also required. Sticking also affects the cloud nucleating properties of these particles and so their exact nature and temporal development is important.

Finally, the radiative properties of rocket plume particulate are not fully quantified and certainly the radiative properties as plume and atmospheric species stick to the alumina are not known. This adds to the uncertainty in evaluating the radiative forcing of the plumes on the atmosphere and in turn introduces uncertainties into the climate model calculations.

\subsubsection{Boundary layers}

Due to viscous interaction with the nozzle internal wall a boundary layer develops which is initially laminar but at some point transitions to turbulent. Currently, nozzles can be designed with consideration for the boundary layer using Momentum Integral Methods and/or CFD and can, if required, be designed to accommodate the displacement thickness due to the boundary layer and hence improve the expansion efficiency of the nozzle. However, at the nozzle trailing edge this boundary layer separates and forms a shear layer between the core plume and the external freestream. This shear layer is hotter than both the plume and freestream and has an intermediate velocity. The effect of this layer on the subsequent evolution of the plume can only be assessed using experiments coupled with computations and would rely heavily on the accuracy at which the boundary layer at the nozzle exit plane is characterized.

\subsubsection{Freestream conditions}

The nature of the freestream conditions can affect the development of the plume. Large scale turbulent features can increase the mixing process whilst wind shear can stretch the plume. Meilinger et al. [19] found that the development of the plume depended on the exact nature of the freestream and required statistical averaging of perturbations to the freestream to calculate global emission indexes in the developing plume. There is no reason that this will not also be the case for rocket plumes and any computation of impact requires either temporal atmospheric data or a mean and some standard deviation.

Computations should also reflect the fact that the launcher altitude range is ground to extra atmosphere and the plume analysis should be conducted over a wide range of altitudes.

\subsubsection{Launcher configuration}

The launcher configuration affects the nature of the freestream flow surrounding the plume which in turn impacts the interaction between the two. While the 
mean flow around the vehicle can be modeled using CFD, the unsteady phenomena such as turbulent boundary layer, buffeting and vorticity shedding increase the required complexity and computational resources.

A large influence on the plume development will also be the configuration of boosters. As an example, during the initial $120 \mathrm{~s}$ of the Space Shuttle, H-2A, Ariane and Soyuz trajectories multiple rocket engines are simultaneously firing. The multiple plumes will interact forming a complex flowfield which requires a quite computationally expensive 3D flowfield analysis for a proper assessment. Lohn et al. [20] calculated an enhancement of temperatures in the afterburning region due to the interaction of the Titan IIIC rocket booster plumes and predicted complete conversion of $\mathrm{HCl}$ to $\mathrm{Cl}$. Gates et al. [3] also surmised that some configurations could have a stronger interaction than others.

The impact of one launch vehicle cannot therefore be simply derived from the impact of another launcher and although the U.S. flight campaigns have provided important data, they cannot replace particular flight data when assessing the impact of other launchers on the atmosphere.

\subsection{Low Order Analysis of the Emissions from a Titan IV SRM}

The uncertainty introduced by the phenomenon listed in the previous section can be assessed using the following simple analysis of the Titan IV rocket. Baseline data of the on design nozzle exit conditions is calculated using the NASA CEA equilibrium code [21] and using the rocket function. Two propellant formulations are assessed, with PBAN (Polybutadiene acrylonitrile) or HTPB (HydroxylTerminated Polybutadiene) binders used. The baseline data is compared with a simplified model of afterburning and mixing where the conditions in the combustion chamber are assumed to be the conditions due to afterburning (in effect adiabatic deceleration of the plume to zero velocity). Mixing with the air is then simulated by adding a fraction of the air $\left(\mathrm{N}_{2}+\mathrm{O}_{2}\right)$ to the rocket propellant at $300 \mathrm{~K}$. The results for PBAN are shown in Table 1 and HTPB in Table 2.

Table 1 Assessment of the effect of afterburning and mixing on the plume conditions of a Titan IV, PBAN (approximated as 68.5\% Ammonium Perchlorate, 15.5\% aluminum, and $16 \%$ PBAN $\left.\left(\mathrm{C}_{10} \mathrm{H}_{13} \mathrm{NO}_{2}\right)\right)$ rocket motor. The data are given as a ratio between the mass of a species to the mass of propellant. Other species are not included. Fractions in the second scenario sum to 2 since there is $2 \mathrm{~kg}$ of mixture for each kilogram of propellant

\begin{tabular}{lcccccccc}
\hline \multicolumn{1}{c}{ Scenario } & $\mathrm{Cl}$ & $\mathrm{HCl}$ & $\mathrm{CO}$ & $\mathrm{CO}_{2}$ & $\mathrm{OH}$ & $\mathrm{NO}$ & $\mathrm{Al}_{2} \mathrm{O}_{3}$ & $\mathrm{H}_{2} \mathrm{O}$ \\
\hline Baseline & 0.004 & 0.208 & 0.220 & 0.048 & 0 & 0 & 0.294 & 0.115 \\
\hline $\begin{array}{l}\text { Afterburning + mixing } \\
\text { with equal mass of air }\end{array}$ & 0.033 & 0.176 & 0.116 & 0.211 & 0.027 & 0.025 & 0.290 & 0.227 \\
\hline
\end{tabular}


Table 2 Assessment of the effect of afterburning and mixing on the plume conditions of a Titan IV, HTPB (approximated as 70\% Ammonium Perchlorate, 18.5\% aluminum, and $11.5 \%$ HTPB $\left.\left(\mathrm{C}_{4} \mathrm{H}_{6}\right)\right)$ ) rocket motor

\begin{tabular}{lcccccccc}
\hline \multicolumn{1}{c}{ Scenario } & $\mathrm{Cl}$ & $\mathrm{HCL}$ & $\mathrm{CO}$ & $\mathrm{CO}_{2}$ & $\mathrm{OH}$ & $\mathrm{NO}$ & $\mathrm{Al}_{2} \mathrm{O}_{3}$ & $\mathrm{H}_{2} \mathrm{O}$ \\
\hline Baseline & 0.003 & 0.214 & 0.222 & 0.0257 & 0 & 0 & 0.348 & 0.081 \\
\hline $\begin{array}{l}\text { Afterburning + mixing } \\
\text { with equal mass of air }\end{array}$ & 0.032 & 0.180 & 0.141 & 0.1520 & 0.026 & 0.02 & 0.345 & 0.222 \\
\hline
\end{tabular}

The baseline data for HTPB agrees within $5 \%$ of that given in [1] while the PBAN data differs by up to $50 \%$ in the $\mathrm{CO}, \mathrm{CO}_{2}$ and $\mathrm{H}_{2} \mathrm{O}$ species fractions. However, there was found a considerable dependency on the exact nature of the PBAN mass fractions which had to be assumed, demonstrating a need for an accurate fuel description.

This simple analysis demonstrates some important points:

- the baseline data does not agree with findings in the literature since $\mathrm{Cl}_{x}$ fractions are far too low, as are levels of $\mathrm{CO}_{2}, \mathrm{OH}$ and NO. Clearly, afterburning has an important role in increase of the levels of ozone depleting species in the plume whilst mixing with air oxidises the species increasing the levels of Greenhouse Gases;

- for both propellant formulations, the afterburning process increases the partitioning of $\mathrm{Cl}$ from $\mathrm{HCl}$. However, this increase is not consistent with flight campaigns described by Ross et al. [22, 23] who measured over $30 \%$ of $\mathrm{HCl}$ conversion to $\mathrm{Cl}_{2}$ in the wake of the TITAN launcher. More complete plume models used in the Ross study were more capable in capturing this increase in $\mathrm{Cl}$, but errors persisted. Exact levels of $\mathrm{Cl}$ and $\mathrm{Cl}_{2}$ in the plume are important as $\mathrm{Cl}$ is a vigorous ozone depleter as a catalyst and, therefore, is of significant importance to climate modeling;

- $\mathrm{CO}$ oxidises to $\mathrm{CO}_{2}$ (Greenhouse Gas), a phenomenon seen in the U.S. flight campaigns, but not to the degree seen in the literature. The data from [3] show that the level of $\mathrm{CO}_{2}$ is dependant on the launcher but is typically correlated to and higher than the mass fraction of alumina in the plume. The correlation implies that heterogeneous chemistry is occurring in the plume and should be modeled; and

- the increase of NO (Ozone Depleter) and OH from trace species to 1\%-2\% of the exhaust mass is consistent with the data from Popp et al. [4] who measured $\mathrm{NO}_{y}$ levels of $2.7 \%$ of the fuel mass in the plume of an Athena II rocket.

The results show that the qualitative effects of afterburning and mixing in the plume can be determined using lower order methods but the uncertainty 
involved in quantitative data is large. More accurate plume models are required. These plume models will require validation for processes such as heterogeneous reactions and the plume development. The required work is discussed later.

\section{UNCERTAINTY IN CLIMATE MODELING}

\subsection{Overview}

The residence time of gas and particles released in the middle atmosphere is very long, typically of the order of several years. It is in contrast with the troposphere where, apart from $\mathrm{CO}_{2}$, most of the compounds (gas, particles) released by rockets are removed within less than a week. In addition, almost all the gases and particles are either chemically active, or climate radiatively active or both. Depending on the nature of the rocket propellant, rockets can release potentially ozone-destroying compounds (chlorine, water vapor, nitrogen oxides, etc.), chemically and optically active particles (aluminum oxides for SRM, soot and sulphuric acid particles for kerosene etc.) that might also act as cloud condensation nuclei $(\mathrm{CN})$, and gaseous cloud precursors (water vapor, nitrogen oxides being converted to nitric acid). It is therefore necessary that an accurate inventory of emitted species be compiled and their evolution within a dynamically and chemically active plume be described.

Although rockets emit several compounds that can interact with the solar and terrestrial radiation, little attention has been paid to the effects of rockets on the radiative balance of the atmosphere. It is in contrast to the abundant literature on the impact of rocket launches on stratospheric ozone [6, 24-27]. The only exception is a recent study [28] on the impact of possible future space tourism on the stratospheric soot loading and the associated climate forcing.

Although it is expected to be very small, there has not been a reliable quantification of the impact of present-day rocket launches on the climate. It should be possible to estimate the direct radiative forcing of rocket emissions of greenhouse gases such as $\mathrm{CO}_{2}$ and $\mathrm{H}_{2} \mathrm{O}$ because climate assessments for such gases are rather common. It would be much more difficult to carry out such a climate evaluation for aluminum oxide particles because their optical properties are very poorly characterised. Note also that their heterogeneous chemical reactivity is also poorly known. Indirect forcing from for example ozone changes caused by rocket launches need also to be considered if the climate assessment is to be complete.

Uncertainties occur during climate modeling due a number of reasons. As with most numerical approaches, the accuracy of the model depends on the complexity of the numerics implemented and the level of inclusion of physical phenomenon. Climate models may differ by the way they discretize and resolve equations driving the chemistry-climate system, by their spatial domains, grids and resolutions, by their parameterizations of sub-grid scale physical processes 
(radiative transfer, cloud formation, convection,... ), and by their choices of input parameters, etc. However, more specifically to the case of plume impact on the atmosphere, uncertainty is also due to the method by which the plume is implemented in a climate model and also the scalability between the real and modeled scenarios. These two issues are expanded upon in the following sections.

\subsection{Implementing the Plume into Climate Models}

In order to be able to calculate the climate effects of rocket plumes, one has to describe their formation and evolution in global climate models. As it stands, global climate models cannot resolve plumes because of their coarse resolution. Like clouds, rocket plumes have to be parametrised on a subgrid scale level. The design and development of such specific parametrisation is not straightforward. It is particularly the case when the represented subgrid scale processes are highly nonlinear and when the parametrisations are to be as physically based as possible in order to account in a realistic way for the effects of large-scale dynamics on subgrid scale fields. Developing subgrid scale parametrisations for large-scale models usually relies on small scale resolving simulations that are carried out at high resolutions and on identifying simple relationships that could describe how, for example, rocket plumes evolve with environmental conditions. The dispersion of rocket plumes depends, among other factors, on atmospheric conditions. Static stability and wind shear determine to what extent plumes are stretched and ultimately mixed with the surrounding air. Parameterisations would ideally account for these effects.

Dynamical processes are not the only nonlinear processes relevant to rocket plumes. Chemical processes occurring within the plume, especially the ozonedestroying chemistry, are also typically nonlinear. The parameterisations would also have to account for these nonlinearities. Indeed, rocket emissions are very localised, producing a chemistry that is quite different from what would operate if the compounds were emitted in the large grid boxes of global models.

In effect, the plume processes outlined in the previous section should be computed in models where they can be resolved up to a scale when they are sufficiently developed for interpolation and parametrisation onto the climate model subgrid scale (it is not known a priori if this can be done) or even developed to size greater than the subgrid scale and consequently can be directly used as input into the large-scale climate models without interpolation.

\subsection{Scalability}

Another important aspect of the rocket problem is the scalability of launches. It is not clear at this point whether the results from a climate modeling study of one rocket launch can be extrapolated to the real situation where approximately 100 launches occur per year. Ross et al. [29] noted that by assuming that 
rocket launch impacts on ozone were linearly cumulative they ignored possibly important second order effects. These second-order effects can be seen as contributing to a cumulative atmospheric perturbation which should be considered in the background atmosphere for subsequent launches. For example, one can imagine the scenario where two vehicles launch within a very short time span, short enough that the local atmosphere between launches has not recovered to the baseline atmosphere before either launch. In this case, the change in atmospheric conditions will of course need to be taken into account when running the computation for the second launch. Such second-order effects include: time between launches; total number of launches; launch vehicle configuration; launch site; and cumulative atmospheric perturbation.

\section{REQUIRED FUTURE WORK}

Assuming the goal of assessing the atmospheric effects of a particular launch or launch program, a weakly coupled aerothermodynamics and climate simulation is required. At this point, weak coupling is appropriate providing that a range of rocket plume conditions (mean conditions, spread and errors) are used as input into the climate modeling. A number of climate simulations can then be run using statistical methods such as Monte Carlo to assess the effect of the uncertainties in aerothermodynamic conditions on the global impact. This would result in an impact assessment with error bars, the size of which depends on the uncertainties included in the input data and the inherent uncertainties in climate models. More accurate data on the plume conditions will be required if the assessment of the impact is to be meaningful and reliable. For this, the following is required.

\subsection{Validation Data}

The most important aspect of any simulation study is the level to which the computations can be validated. This naturally depends on the quality of the reference validation data. Rocket plumes by their nature are very difficult to validate on ground as they are hot, reactive, large scale and interacting with a supersonic low density environment. Currently, Europe does not have a fully inclusive hot plume testing wind tunnel with supersonic freestream capability. Despite this, some significant steps can be taken to provide high level validation data.

\subsubsection{Laboratory experiments}

Alumina is a product of the combustion of solid boosters. They are formed in the hot combustor but quickly solidify during the fast expansion in the rocket 
nozzle. Anecdotal evidence has shown that it may affect the chemistry of the plume by participating in heterogeneous reactions and, therefore, changing the partition of species fractions [30]. For example, it is thought that $\mathrm{HCl}$ can be converted into chlorine ozone-destroying species on alumina. If this mechanism is correct, this would increase ozone depletion.

Currently, there are large uncertainties and unknowns pertaining to sticking coefficients and reaction probabilities of chemicals onto alumina. This affects the ability to predict heterogeneous chemistry, the life span of reactive particles (over time, the particle may age and become inert due to coating by other substances) and the capability of the particle to act as a cloud CN. Furthermore, the calculation of the radiative forcing of alumina requires detailed information about the absorption and scattering (forward and backward) of alumina particles, both pure, with other chemicals bonded to the surface and mixed with sulphuric acid aerosol particles for example. The following data should be characterized in laboratory conditions:

- precise reactions rates mechanism of alumina within plume conditions;

- sticking coefficients of plume species onto alumina particles and their variations with particle properties as the plume develops; and

- the radiative properties of the pure, modified (due to sticking etc.), and mixed alumina particle.

\subsubsection{On ground rocket testing}

The conditions of rockets at the nozzle exit plane can be better understood with ground testing. The simulation of the rocket chamber is complicated by the vigorous combustion process, the expansion process and the presence of boundary layers and instabilities. Solid rocket motors have the added complexity of alumina particulate. Current rocket test bench facilities should provide detailed data on the chemical nature of the exhaust plane using spectroscopy techniques.

Currently, alumina particulate can be modeled within a nozzle and plume using CFD with Lagrangian tracking but there is no data to validate the results. An experiment is required to identify the size distribution, velocity, and space distribution of alumina at a particular inlet plane, an obvious place being the throat, and then to track the development of the particles through the nozzle. High capture rate cameras are reliably used for similar circumstances as part of PIV measurement systems. Further, the algorithms used in these velocity measurement systems can be reused for assessing the particulate velocity while further algorithms would be required to track the development of the particle size (if they change) and distribution. At this point, a simple test bed would be required with the inlet conditions not necessarily matching a real rocket engine but with representative and accurate boundary conditions. 


\subsubsection{Flight tests}

Ground tests and laboratory experiments will not capture the full dynamics of a real plume in the atmosphere, particularly when a hot plume wind tunnel is available. Flight tests will then be required to provide data on the temporal and spatial development of the plume. Ideally, this would consist of two measurement positions:

(1) on board the launch vehicle: dedicated measurement devices could be placed onboard the launch vehicle and measuring some of the conditions of the early plume. This, however, would affect the characteristics of the carefully designed launch vehicle and may be unreasonable. However, it is worthwhile investigating the possibility as it would provide unique data on the initial plume under real conditions; and

(2) through the developing plume: as mentioned earlier, it is not possible at this point to accurately interpolate the plume data from U.S. tests to other launch vehicles. Therefore, a dedicated flight test through each plume is required.

\subsection{Climate Simulations}

Before considering large-scale climate simulations, the first step would be to simulate the spatio-temporal evolution of the plume chemical composition and conditions. The small-scale plume simulations would need to be evaluated against available data and observations. Then, these small-scale simulations would be used to develop subgrid-scale parametrisations for large-scale climate models. Aside from the obvious validation exercises required for computations, further development may be required once deficiencies in models or assumptions become known. Considering the number of areas where uncertainty occurs, it would be useful to have some knowledge of which parameters have the greatest impact on the variance of the final impact figure. Therefore a series of climate simulations, coupled with statistical methods such as Monte Carlo simulations and regression analysis should be undertaken to provide the sensitivity of impact to each of the parameters (such as $\mathrm{Cl}$ levels, scalability, mixing etc.).

\section{CONCLUDING REMARKS}

The previous sections have briefly presented some of the areas of uncertainty when it comes to modelling or assessing the effects of rocket launches on the atmosphere. It should be clear that any plume or climate model used for such a task requires careful validation to assess its accuracy. The U.S. has taken steps to validate codes by undertaking flight campaigns through the plumes of its rocket 
fleet, yet plume models still give inconsistent results. This implies a need for further flight and ground testing of the various points of ambiguity illustrated above.

Clearly, the processes outlined above have strong effects on the overall plume chemistry and evolution, and plume models should be validated that they properly include these processes. Validation using ground testing would require an engine firing in representative wind tunnel conditions. Although ESA is investigating the possibility of such a facility, it is currently not available in Europe. Vehicle to vehicle flight data cannot be compared, limiting the value of the U.S. flight data for extrapolation to other launchers.

Any reductions in the uncertainty associated with the effects that rocket plumes have on the atmosphere would reduce the error associated with any calculation of impact and therefore reduce the maximum impact estimate, thus leading to an increase in ESA's understanding of the actual effects of launchers on the environment.

\section{REFERENCES}

1. NASA. 1995. Final environmental impact statement for the Cassini mission. http://saturn.jpl.nasa.gov/spacecraft/safety/safetyeis/.

2. Cziczo, D. J., D. M. Murphy, D. S. Thomson, and M. N. Ross. 2002. Composition of individual particles in the wakes of an Athena II rocket and the space shuttle. Geophys. Res. Lett. 29:2037.

3. Gates, A. M., L. A. Avallone, D. W. Toohey, et al. 2002. In situ measurements of carbon dioxide, $0.37-4.0 \mu \mathrm{m}$ particles, and water vapour in the stratospheric plumes of small rockets. J. Geophys. Res. 107(D22):4649.

4. Popp, P. J., B. A. Ridley, J. A. Neuman, et al. 2002. The emission and chemistry of reactive nitrogen species in the plume of an Athena II solid-fueled rocket motor. Geophys. Res. Lett. 29(18):1887.

5. Schmid, O., J. M. Reeves, J. C. Wilson, et al. 2003. Size-resolved particle emission indices in the stratospheric plume of an Athena II rocket. J. Geophys. Res. 108:4250.

6. Jackman, C. H., D. B. Considine, and E. L. Fleming. 1996. Space shuttle's impact on the stratosphere: An update. J. Geophys. Res. 101(D7):12,523.

7. Siskind, D. E., M. H. Stevens, J. T. Emmert, et al. 2003. Signatures of shuttle and rocket exhaust plumes in TIMED/SABER radiance data. Geophys. Res. Lett. $30: 1819$.

8. Stevens, M.H., R.R. Meier, X. Chu, et al. 2005. Antarctic mesospheric clouds formed from space shuttle exhaust. Geophys. Res. Lett. 32:L13810.

9. Sutton, G., and O. Biblarz. 2001. Rocket propulsion elements. John Wiley and Sons.

10. Tsimis, C. 2007. Fuel jet injection and supersonic mixing for scramjets. Ph.D. Thesis. Imperial College London.

11. Papamoschou, D. 1989. Structure of the compressible turbulent shear layer. AIAA Paper No. 89-0126.

12. Gutmark, E., K. Schadow, and K. Wilson. 1990. Effect of convective Mach number on mixing of coaxial circular and rectangular jets. Phys. Fluids A 3(1):29. 
13. Heinza, S. 2003. A model for the reduction of the turbulent energy redistribution by compressibility. Phys. Fluids 15(11):3580.

14. Brady, B., and R. Martin. 1996. Modelling solid rocket booster exhaust plumes in the stratosphere with SURFACE CHEMKIN. Aerospace Report No. TR95(5231)-9.

15. Rosario, N. 2011. Simulation of the propulsive system of a rocket. Masters Thesis. Instituto Superior Técnico/ESA Internal Report.

16. Ross, M., D. W. Toohey, W. T. Rawlins, et al. 2000. Observation of stratospheric ozone depletion associated with Delta II rocket emissions. Geophys. Res. Lett. 27(15):2209-12.

17. Bekki, S. 1997. On the possible role of aircraft-generated soot in the middle latitude ozone depletion. J. Geophys Res. 102(D9):10,751.

18. Sander, S. P., J. Abbatt, J. R. Barker, J. B. Burkholder, R. R. Friedl, D. M. Golden, R. E. Huie, C. E. Kolb, M. J. Kurylo, G. K. Moortgat, V. L. Orkin, and P. H. Wine. 2011. Chemical kinetics and photochemical data for use in atmospheric studies. Evaluation No.17. JPL Publication 10-6. Jet Propulsion Laboratory, Pasadena. http://jpldataeval.jpl.nasa.gov.

19. Meilinger, S., B. Kircher, and Th. Peter. 2005. Microphysics and heterogeneous chemistry in aircraft plumes - high sensitivity on local meteorology and atmospheric composition. Atmos. Chem. Phys. 5:533-45.

20. Lohn, P., E. Wong, T. Smith, J. Edwards, and D. Pilsen. 1999. Rocket exhaust impact on stratospheric ozone. Prepared for U.S. Air Force Space and Missile Systems Center, Environmental Management Branch, SMC/AXFV.

21. Gordon, S., and B. J. McBride. 1996. Computer program for calculation of complex chemical equilibrium compositions and applications. NASA Reference Publication 1311.

22. Ross, M. N., J. O. Ballenthin, R. B. Gosselin, et al. 1997. In-situ measurement of $\mathrm{Cl}_{2}$ and $\mathrm{O}_{3}$ in a stratospheric solid rocket motor exhaust plume. Geophys. Res. Lett. 24(14):1755.

23. Ross, M., and P. Zittel. 2000. Rockets and the ozone layer. Crosslink - The Aerospace Corporation Magazine of Advances in Aerospace Technology 1(2).

24. Jones, A. E., S. Bekki, and J. A. Pyle. 1995. On the atmospheric impact of launching the Ariane 5 rocket. J. Geophys. Res. 100(D8):16,651.

25. Jackman, C. H., D. B. Considine, and E. L. Fleming. 1998. A global modeling study of solid rocket aluminum oxide emission effects on stratospheric ozone. J. Geophys. Res. Lett. 25(6):907.

26. Danilin, M. Y., M. K. W. Ko, and D. K. Weisenstein. 2001. Global implications of ozone loss in a space shuttle wake. J. Geophys. Res. 106(D4):3591.

27. Danilin, M.Y., R.-L. Shia, M. K. W. Ko, et al. 2001. Global stratospheric effects of the alumina emissions by solid-fueled rocket motors. J. Geophys. Res. 106(D12):12,727.

28. Ross, M. N., M. Mills, and D. Toohey. 2010. Potential climate impact of black carbon emitted by rockets. Geophys. Res. Lett. 37:L24810.

29. Ross, M., D. Toohey, M. Peinemann, and P. Ross. 2009. Limits on the space launch market related to stratospheric ozone depletion. Astropolitics 7:1, 50-82.

30. Molina, M., L. Molina, R. Zhang, R. Meads, and D. Spencer. 1997. The reaction of $\mathrm{ClONO}_{2}$ with HCL on alumina oxide. J. Geophys. Res. 24(13):1619. 